\title{
Caspofungin Exposure Alters the Core Septin AspB Interactome of Aspergillus fumigatus
}

José M. Vargas-Muñiz ${ }^{1}$, Hilary Renshaw ${ }^{1}$, Greg Waitt ${ }^{2}$, Erik J. Soderblom ${ }^{2}$, M. Arthur Moseley ${ }^{2}$,

Jonathan M. Palmer ${ }^{3,5}$, Praveen R. Juvvadi ${ }^{4}$, Nancy P. Keller ${ }^{5}$, William J. Steinbach ${ }^{1,4 \#}$ 


\section{Abstract}

28 Aspergillus fumigatus, the main etiological agent of invasive aspergillosis, is a leading cause of

29 death in immunocompromised patients. Septins, a conserved family of GTP-binding proteins,

30 serve as scaffolding proteins to recruit enzymes and key regulators to different cellular

31 compartments. Deletion of the A. fumigatus septin aspB increases susceptibility to the

32 echinocandin antifungal caspofungin. However, how AspB mediates this response to

33 caspofungin is unknown. Here, we characterized the AspB interactome under basal conditions

34 and after exposure to a clinically relevant concentration of caspofungin. While A. fumigatus

35 AspB interacted with 334 proteins, including kinases, cell cycle regulators, and cell wall

36 synthesis-related proteins under basal growth conditions, caspofungin exposure altered AspB

37 interactions. A total of 69 of the basal interactants did not interact with AspB after exposure to

38 caspofungin, and 54 new interactants were identified following caspofungin exposure. We

39 generated A. fumigatus deletion strains for 3 proteins (ArpB, Cyp4, and PpoA) that only

40 interacted with AspB following exposure to caspofungin that were previously annotated as

41 induced after exposure to antifungal agents, yet only PpoA was implicated in the response to

42 caspofungin. Taken together, we defined how the septin AspB interactome is altered in the

43 presence of a clinically relevant antifungal.

44

45 Key words: Aspergillus fumigatus, septins, AspB, caspofungin, interactome 


\section{Introduction}

Aspergillus fumigatus, the main etiological agent of invasive aspergillosis, is a leading

51 cause of fungal mortality in immunocompromised patients [1, 2]. Although the incidence of

52 invasive aspergillosis has increased in the last two decades due to a rise in the

53 immunocompromised patient population, there is a lack of effective treatments and basic

54 understanding of growth and disease [3]. The echinocandin caspofungin is guideline-

55 recommended as a second-line therapy for invasive aspergillosis [4]. With the increasing

56 emergence of azole resistance amongst $A$. fumigatus isolates, the echinocandins are increasingly

57 used to treat invasive aspergillosis and it is therefore critical to understand the fungal response to

58 these antifungal agents [5]. Caspofungin targets $\beta-1,3-\mathrm{D}$-glucan synthase, which synthesizes a

59 major component of the A. fumigatus cell wall $[6,7]$. Treatment with caspofungin is fungistatic

60 against $A$. fumigatus, yet the mechanism behind the A. fumigatus response to this commonly-

61 used antifungal is not fully understood [7-9].

Septins are conserved GTPases that are involved in a myriad of cellular processes,

63 ranging from cytokinesis to cell morphology [10-15]. Septins have been previously linked to cell

64 wall biogenesis in Saccharomyces cerevisiae, where they are required for the proper localization

65 of chitin synthase [16]. Deletion or mutation of septin genes lead to an increase in the

66 susceptibility to caspofungin in Candida albicans and A. fumigatus [17, 18]. These fungal septins

67 re-localize after caspofungin exposure, suggesting a role for septins in response to this

68 echinocandin $[18,19]$. However, the mechanism behind the septin-mediated response to

69 caspofungin remains unknown. In order to further understand how AspB contributes to this

70 caspofungin response, we analyzed the AspB protein interactome under both basal conditions

71 and following exposure to a clinically relevant concentration of the antifungal caspofungin. 


\subsection{Strain, media, and culture conditions}

74 Cultures were grown on glucose minimal media (GMM) at $37^{\circ} \mathrm{C}$, except where otherwise

75 specified. Escherichia coli $\mathrm{DH} 5 \alpha$ competent cells were used for cloning. The aspB-egfp nimX-

$76 r f p$ strain was generated by cloning $1 \mathrm{~kb}$ of $\operatorname{nim} X$ and the terminator sequence into the pJW24-

77 RFP-NS vector. The resulting plasmid was digested with NotI and SalI and transformed into the

$78 a k u B^{\mathrm{KU} 80, \text { pyrG- }} \quad \operatorname{aspB-egfp}$ strain [20]. Deletion of the arp2 ortholog, which we named $\operatorname{arpB}$

79 due to a gene involved in melanin biosynthesis sharing the arp2 name [21], was attained by

80 replacing the $1.6 \mathrm{~kb}$ arpB (Afu1g13330) with the $3.0 \mathrm{~kb}$ Aspergillus parasiticus pyrG cassette.

81 Approximately $1 \mathrm{~kb}$ of upstream and downstream flanking regions of arpB were PCR-amplified

82 from AF293 genomic DNA and cloned into the pJW24 plasmid. The resulting plasmid was

83 digested with NotI and SalI and transformed into the $a k u B^{\mathrm{KU} 80} p y r G^{-}$strain. Deletion of $c y p 4$ was

84 performed by similarly replacing the $0.8 \mathrm{~kb}$ cyp4 (Afu2g03720) with the $3.0 \mathrm{~kb}$ A. parasiticus

85 pyrG cassette. The resulting plasmid was digested with NotI and SalI and transformed also into

86 the $a k u B^{\mathrm{KU} 80} \operatorname{pyrG}^{-}$strain. The $\triangle p p o A$ strain has been previously described [22] and the

87 OE::ppoA strain was constructed in the AF293.1 background [23] through promoter replacement

88 with a fusion PCR product [24] consisting of $\sim 1 \mathrm{~kb}$ flanking regions around the A. parasiticus

89 pyrG::A. nidulans gpdA promoter cassette from pJMP9 [25]. Strains generated in this study were

90 confirmed by PCR (data not shown) and Southern blotting (Fig. S1). The aspB-egfp ppoA-rfp

91 strain was generated by cloning $1 \mathrm{~kb}$ of $p p o A$ and terminator sequence into the pJW24-RFP-NS

92 vector. The resulting plasmid was digested with NotI and SalI and transformed into the $a k u B^{\mathrm{KU} 80 \text {, }}$

93 pyrG- $a s p B-e g f p$ strain (Table $\mathrm{S} 1)$. 
95 The A. fumigatus strain expressing the aspB-egfp fusion construct under the control of the aspB 96 native promoter was grown in GMM liquid media and GMM liquid media supplemented with 1

$97 \mu \mathrm{g} / \mathrm{mL}$ of caspofungin for $24 \mathrm{~h}$ at $37^{\circ} \mathrm{C}$. Total cell lysate was obtained by homogenizing the

98 fungal mycelia (1 g wet weight) as previously described [26]. Total protein from the crude

99 extract was quantified by the Bradford method and purified using the GFP-Trap ${ }^{\circledR}$ affinity 100 purification (Chromotek) as previously described [26].

101 Resin-bound proteins were then reduced with $5 \mathrm{mM}$ dithiothreitol for $30 \mathrm{~min}$ at $37^{\circ} \mathrm{C}$ and 102 alkylated with $12 \mathrm{mM}$ iodoacetamide for $45 \mathrm{~min}$ at room temperature. Trypsin digestion was 103 allowed to proceed on-resin for $18 \mathrm{~h}$ at $37^{\circ} \mathrm{C}$. Samples were then subjected to a $90 \mathrm{~min}$ 104 chromatographic separation on a Waters NanoAquity UPLC equipped with a $1.7 \mu$ m BEH130 $105 \mathrm{C}_{18} 75 \mu \mathrm{m}$ I.D. X $250 \mathrm{~mm}$ reversed-phase column as previously described [26]. The analytical 106 column was coupled to a Thermo QExactive Plus high-resolution mass spectrometer through an 107 electrospray ionization source. The instrument was operated in a data-dependent mode of 108 acquisition with a precursor MS scan from m/z 375-1600 at $\mathrm{r}=70,000$ followed by ten MS/MS 109 spectra at $\mathrm{r}=15,000$ using a $26 \%$ CID energy setting.

110 Mass spectra were processed with Mascot Distiller (Matrix Science) and were then submitted to 111 Mascot searches (Matrix Science) against an NCBI_RefSeq_Aspergillus database at 5 ppm 112 precursor and 0.02 Da product ion mass tolerances. Static mass modifications corresponding to 113 carbamidomethylation on Cys residues and dynamic mass modifications corresponding to 114 oxidation on Met residues and deamidation on Asn and Gln residues were included. Searched 115 spectra were imported into Scaffold v4.3 (Proteome Software) and scoring thresholds were set to 
116 yield a $0.1 \%$ protein false discovery rate. A minimum of two unique peptides from each protein

117 was required for identification.

\section{2.3. Antifungal susceptibility testing}

119 To determine the effect of antifungal agents on the three AspB interactant deletion strains

$120(\triangle p p o A, \triangle c y p 4$ and $\triangle a r p B)$ as well as the $p p o A$ overexpression strain $(O E:: p p o A), 10^{4}$ conidia

121 from these strains and the AF293 control (wild-type) strain were inoculated on GMM agar 122 supplemented with either caspofungin $(1 \mu \mathrm{g} / \mathrm{mL})$ or nikkomycin $\mathrm{Z}(2 \mu \mathrm{g} / \mathrm{mL})$ and growth 123 visualized after 3 days of incubation at $37^{\circ} \mathrm{C}$. Antifungal susceptibility testing was also 124 performed following standard CLSI criteria [27] and the minimal effective concentration (MEC) 125 of the anti-cell wall agents determined for each strain [28].

\section{2.4. Fluorescence microscopy}

127 Conidia $\left(10^{4}\right)$ of the AspB-EGFP NimX-RFP or AspB-EGFP PpoA-RFP strain were cultured on 128 coverslips immersed in $10 \mathrm{~mL}$ of GMM+UU broth and incubated for $20 \mathrm{~h}$ at $37^{\circ} \mathrm{C}$, as previously 129 described [29]. To examine co-localization after exposure to anti-cell wall agents, caspofungin (1 $130 \mu \mathrm{g} / \mathrm{mL}$ ) was added after $18 \mathrm{~h}$ of incubation and samples imaged after $2 \mathrm{~h}$ following drug co131 incubation at $37^{\circ} \mathrm{C}$. Localization patterns were visualized using an Axioskop 2 plus microscope 132 (Zeiss) equipped with AxioVision 4.6 imaging software.

\section{3. Results}

134 3.1. AspB interacts with the septin complex, cytoskeleton, and cell-cycle regulatory proteins

135 Previously, the septin AspB has been shown to interact with other members of the septin 136 family in Aspergillus fumigatus and Aspergillus nidulans $[18,30]$. AspB was purified from a 
137 strain expressing the $a s p B-e g f p$ construct under the control of its native promoter using a GFP-

138 Trap® affinity matrix and the AspB bound protein complex subjected to LC-MS/MS analysis for

139 the identification of interactants [18]. A total of 334 proteins co-purified with AspB, including all

140 of the other four A. fumigatus septins (Table 1) (Fig. 1A). In addition to the septin complex,

141 AspB co-purified with cytoskeletal proteins: actin, tubulin (alpha-1, beta subunit, and TubB

142 subunit), and Arp 2/3 complex components. Previously identified septin-interacting proteins

143 from $S$. cerevisiae and C. albicans, such as Cla4 and $\mathrm{NimX} \mathrm{Xdc}^{\mathrm{C} 8}$, were also co-purified with

144 AspB in A. fumigatus, indicating that septin interactions are somewhat conserved across different

145 fungal species [31-33]. A strain co-expressing nimX-rfp and the aspB-egfp construct under the

146 control of their respective native promoters showed that $\mathrm{Nim} \mathrm{X}^{\mathrm{Cdc} 28}$ colocalized with $65 \%$ of the

147 AspB double bars observed (Fig. S2), validating its interaction with AspB.

\subsection{AspB protein interactions are altered following caspofungin exposure}

Previously, septins have been implicated in response to cell wall stress in different fungal

150 species $[18,19]$. In A. fumigatus, the AspB localization pattern is altered after exposure to anti-

151 cell wall agents nikkomycin $\mathrm{Z}$ and caspofungin [18]. Furthermore, deletion of aspB increases the 152 susceptibility of $A$. fumigatus to these anti-cell wall agents. However, how AspB contributes to

153 the response to anti-cell wall agents remains elusive. We grew the $a s p B$-egfp expressing strain in

154 the presence and absence of caspofungin to define this differential protein interactome.

155 Following caspofungin exposure, 265 proteins continued to be pulled down with AspB-GFP and

15669 proteins no longer co-purified under this condition (Fig. 1B). Proteins involved in hyphal

157 growth and polarity, such as Cla4, MobB, and RasB, no longer co-purified with AspB. An

158 additional 54 proteins co-purified with AspB only following exposure to caspofungin. Most of 
159 these proteins are involved in metabolism and secondary metabolism (two proteins involved in

160 pseurotin A production and one uncharacterized synthase) (Table 2)[34].

161 3.3. Deletion of ppoA results in increased susceptibility to caspofungin in liquid media

162 In order to understand how $\mathrm{AspB}$ might regulate the response to caspofungin, we sought

163 to analyze proteins that only co-purified with AspB after caspofungin exposure and that have

164 been curated to be involved in response to antifungal exposure according to the Aspergillus

165 Genome database (http://www.aspergillusgenome.org/). From the list of interactants, we selected

166 ArpB (an Arp2 ortholog and member of the putative Arp 2/3 complex), Cyp4 (a putative

167 cyclophilin), and PpoA (a characterized fatty acid oxygenase [22, 35]) as prototypical

168 interactants worthy of further exploration (Table 2). Deletion of cyp4 resulted in a reduction in

169 radial growth under basal conditions compared to the wild-type strain (Fig. S3A); however, there

170 was no significant difference in radial growth response to either caspofungin or nikkomycin $\mathrm{Z}$

171 exposure (Fig. S3B). Deletion of ppoA resulted in an increased susceptibility to nikkomycin Z,

172 but not caspofungin, when screened on GMM agar (Fig 2). Nonetheless, the caspofungin

173 minimum effective concentration (MEC) of the $\triangle p p o A$ strain was $0.25 \mu \mathrm{g} / \mathrm{mL}$, compared to the

174 wild-type strain MEC of $1 \mu \mathrm{g} / \mathrm{mL}$ (Table 3). Using a ppoA-rfp aspB-egfp co-expressing strain,

175 we did not observe any AspB and PpoA co-localization under basal conditions or after exposure

176 to anti-cell wall drugs (data not shown). Unfortunately, we were unable to assess ArpB's role in

177 the caspofungin response as we only were able to obtain an $\operatorname{arp} B$ heterokaryon that produced

178 inviable spores, as validated via both germination assays and viability staining with Bis-(1,3-

179 dibutylbarbituric acid) trimethine oxonol (DiBAC) fluorescent dye (data not shown).

\section{Discussion}


182 cytokinesis, hyphal morphology, diffusion barriers, and cell wall integrity [10-13, 15, 36, 37]. In

183 Cryptococcus neoformans, deletion of septin genes leads to an increased susceptibility to cell

184 wall damaging agents such as SDS and caffeine [11]. In C. albicans and A. fumigatus, the core-

185 septin mutants exhibit increased susceptibility to caspofungin [17, 18, 38]. The A. fumigatus

$186 \triangle a s p B$ strain has similar distribution of $\beta$-glucan and chitin when compared to the wild-type

187 strain, and its $\beta$-glucan content was also similar to that of the wild-type strain [18]. However,

188 when the $\triangle a s p B$ strain is exposed to caspofungin, the amount of $\beta$-glucan released increases

189 relative to wild-type strain, indicating aberrant cell wall assembly occurring due to the deletion

190 of $a s p B$ [18]. In C. albicans, exposure to caspofungin leads to a rapid redistribution of septins

191 that co-localize with chitin patches [19]. A. fumigatus AspB also redistributes following exposure

192 to caspofungin, unifying the link between septins and cell wall stress in fungi [18].

193 Here, we explored the AspB interactome under basal conditions as well as after

194 caspofungin exposure to further our understanding of how septins contribute to cell wall integrity

195 in the setting of antifungal exposure with a guideline-recommended clinically approved agent.

196 Echinocandins are increasingly used to treat invasive aspergillosis due to the emergence of azole

197 resistant A. fumigatus isolates [5]. However, there is potential of A. fumigatus isolates to develop 198 resistance to caspofungin [39-41]. Similar to other fungal septins, AspB interacts with the other

199 septins, as well with the actin and microtubule networks [18, 26, 30, 42]. A significant overlap 200 of septin-interacting proteins is observed between the proteins are pulled-down by A. fumigatus

201 AspB and those from the $S$. cerevisiae, suggesting that there is some conservation in protein 202 interaction with septin complexes across fungal species [43]. However, the septin associated 203 kinase Gin4, which associates with the septin complex in S. cerevisiae, did not co-purify with 
204 AspB [43]. It is possible that Gin4 interacts with the A. fumigatus septin complex at a particular

205 stage of growth or cellular process that we failed to capture in our analyses. A. nidulans and $S$.

206 cerevisiae septins' interaction is dependent on the stage of growth, providing further support to

207 this notion $[30,43]$.

The AspB interactome changes following caspofungin exposure, as 69 proteins are no 209 longer co-purified with AspB while 54 new proteins co-purified with AspB under caspofungin 210 exposure. Proteins involved with cell polarity (Cla4, RasB, MobB) no longer interact with AspB, 211 and new metabolically-related proteins interact with AspB after caspofungin exposure, especially 212 those involved in secondary metabolism. In particular, we found two proteins involved in 213 pseurotin A metabolism associated with AspB in these conditions. Pseurotin A is reported to 214 inhibit IgE production [44], and its synthesis is enhanced during hypoxia [45]. However, the role 215 of these metabolism-related proteins in cell wall integrity remains an open question. Of the 216 caspofungin-mediated interactants evaluated, only PpoA is involved in response to the chitin 217 synthase inhibitor nikkomycin $\mathrm{Z}$ when tested on GMM agar. However, we observed an increase 218 in susceptibility of the $\triangle p p o A$ strain to caspofungin when tested via approved antifungal 219 susceptibility methodology. This may suggest that PpoA is involved in some cell wall 220 remodeling process. It is possible that more than one interacting protein contributes to cell wall 221 integrity and they might have overlapping roles; thus, a single gene deletion analysis may not be 222 sufficient to fully define this. In conclusion, AspB interacts with a robust number of proteins that could contribute to 224 the different roles carried out by this septin in both basal growth as well as growth following 225 antifungal stress. After exposure to a clinically relevant concentration of caspofungin, AspB 226 interacts with 54 new proteins, suggesting the importance of AspB in mediating this stress 
227 response. We also observed that in both conditions, members of the canonical cell wall integrity

228 pathway (Rho1, Mkk2, Mpk1) and AspB could serve as a scaffold for signal transduction of the

229 cell wall integrity pathway. The number of spectral counts for Mpk1 doubles compared to the

230 spectral counts of Rho1 and Mkk2 that remain constant. To verify this, further experiments are

231 needed where we can quantitatively compare AspB interaction in the presence and absence of

232 caspofungin, and the role AspB might play in signal transduction of the canonical cell wall

233 integrity pathway.

\section{Acknowledgements}

235 J.M.V. was supported by National Science Foundation Graduate Research Fellowship Program

236 DGF 1106401. W.J.S. and P.R.J. are supported in part by 1 R01 AI112595-01. J.P. and N.P.K.

237 are supported in part by R01-AI065728. We acknowledge Dr. Andrew Alspaugh's laboratory

238 (Duke University) for helpful experimental discussion and technical support. The authors also

239 thank Amber Richards for assisting with some of the experiments. Any opinion, findings, and

240 conclusions expressed in this publication are those of the authors and do not necessarily reflect

241 the views of the National Science Foundation.

\section{Figure Legends}

243 Fig. 1 AspB interactome changes after exposure to a clinically relevant concentration of

244 Caspofungin (A) Biological process gene ontology enrichment of the basal conditions AspB

245 interactome (B) AspB interacts with 54 new proteins after caspofungin exposure.

246 Fig 2. Deletion of ppoA increased susceptibility to nikkomycin Z, but not caspofungin on

247 agar plates. Conidia $\left(10^{4}\right)$ from each strain were inoculated into GMM agar, GMM agar 
248 containing Caspofungin $(1 \mu \mathrm{g} / \mathrm{mL})$ or Nikkomycin $\mathrm{Z}(2 \mu \mathrm{g} / \mathrm{mL})$, and incubated for 3 days at $24937^{\circ} \mathrm{C}$.

250 References:

251 [1] T.R. Dagenais, N.P. Keller, Pathogenesis of Aspergillus fumigatus in Invasive Aspergillosis,

252 Clinical microbiology reviews, 22 (2009) 447-465.

253 [2] G.D. Brown, D.W. Denning, N.A. Gow, S.M. Levitz, M.G. Netea, T.C. White, Hidden

254 killers: human fungal infections, Sci Transl Med, 4 (2012) 165rv113.

255 [3] K.A. Marr, R.A. Carter, F. Crippa, A. Wald, L. Corey, Epidemiology and Outcome of Mould 256 Infections in Hematopoietic Stem Cell Transplant Recipients, Clinical Infectious Diseases (2002) $257909-917$.

258 [4] T.F. Patterson, G.R. Thompson, 3rd, D.W. Denning, J.A. Fishman, S. Hadley, R. Herbrecht, 259 D.P. Kontoyiannis, K.A. Marr, V.A. Morrison, M.H. Nguyen, B.H. Segal, W.J. Steinbach, D.A. 260 Stevens, T.J. Walsh, J.R. Wingard, J.A. Young, J.E. Bennett, Practice Guidelines for the

261 Diagnosis and Management of Aspergillosis: 2016 Update by the Infectious Diseases Society of 262 America, Clinical infectious diseases : an official publication of the Infectious Diseases Society 263 of America, 63 (2016) e1-e60.

264 [5] P.E. Verweij, M. Ananda-Rajah, D. Andes, M.C. Arendrup, R.J. Bruggemann, A.

265 Chowdhary, O.A. Cornely, D.W. Denning, A.H. Groll, K. Izumikawa, B.J. Kullberg, K. Lagrou, 266 J. Maertens, J.F. Meis, P. Newton, I. Page, S. Seyedmousavi, D.C. Sheppard, C. Viscoli, A.

267 Warris, J.P. Donnelly, International expert opinion on the management of infection caused by 268 azole-resistant Aspergillus fumigatus, Drug Resist Updat, 21-22 (2015) 30-40. 
269 [6] M. Bernard, J.P. Latge, Aspergillus fumigatus cell wall: composition and biosynthesis,

270 Medical mycology : official publication of the International Society for Human and Animal

271 Mycology, 39 (2001) 9-17.

272 [7] J.N. Kahn, M.J. Hsu, F. Racine, R. Giacobbe, M. Motyl, Caspofungin susceptibility in

273 Aspergillus and non-Aspergillus molds: inhibition of glucan synthase and reduction of beta-D-1,3

274 glucan levels in culture, Antimicrobial agents and chemotherapy, 50 (2006) 2214-2216.

275 [8] F. Lamoth, P.R. Juvvadi, J.R. Fortwendel, W.J. Steinbach, Heat shock protein 90 is required

276 for conidiation and cell wall integrity in Aspergillus fumigatus, Eukaryotic cell, 11 (2012) 13242771332.

278 [9] L.E. Rogg, J.R. Fortwendel, P.R. Juvvadi, A. Lilley, W.J. Steinbach, The chitin synthase

279 genes chsA and chsC are not required for cell wall stress responses in the human pathogen

280 Aspergillus fumigatus, Biochemical and biophysical research communications, 411 (2011) 549-

281554.

282 [10] Y. Hernandez-Rodriguez, S. Hastings, M. Momany, The septin AspB in Aspergillus

283 nidulans forms bars and filaments and plays roles in growth emergence and conidiation,

284 Eukaryotic cell, 11 (2012) 311-323.

285 [11] L. Kozubowski, J. Heitman, Septins enforce morphogenetic events during sexual

286 reproduction and contribute to virulence of Cryptococcus neoformans, Molecular microbiology,

$287 \quad 75$ (2010) 658-675.

288 [12] L. Li, C. Zhang, J.B. Konopka, A Candida albicans temperature-sensitive cdc12-6 mutant

289 identifies roles for septins in selection of sites of germ tube formation and hyphal

290 morphogenesis, Eukaryotic cell, 11 (2012) 1210-1218. 
291 [13] R. Lindsey, S. Cowden, Y. Hernandez-Rodriguez, M. Momany, Septins AspA and AspC are

292 Important for Normal Development and Limit the Emergence of New Growth Foci in the

293 Multicellular Fungus Aspergillus nidulans, Eukaryotic cell, 9 (2010) 155-163.

294 [14] M. Momany, J. Zhao, R. Lindsey, P.J. Westfall, Characterization of the Aspergillus nidulans

295 Septin (asp) Gene Family, Genetics, 157 (2001).

296 [15] I. Alvarez-Tabares, J. Perez-Martin, Septins from the phytopathogenic fungus Ustilago

297 maydis are required for proper morphogenesis but dispensable for virulence, PloS one, 5 (2010)

298 e12933.

299 [16] D.J. DeMarini, A.E.M. Adams, H. Fares, C. De Virgilio, G. Valle, J.S. Chuang, J.R. Pringle,

300 A Septin-based Hierarchy of Proteins Required for Localized Deposition of Chitin in the

301 Saccharomyces cerevisiae Cell Wall, Journal of Cell Biology, 139 (1997) 1.

302 [17] J.R. Blankenship, S. Fanning, J.J. Hamaker, A.P. Mitchell, An extensive circuitry for cell

303 wall regulation in Candida albicans, PLoS pathogens, 6 (2010) e1000752.

304 [18] J.M. Vargas-Muniz, H. Renshaw, A.D. Richards, F. Lamoth, E.J. Soderblom, M.A.

305 Moseley, P.R. Juvvadi, W.J. Steinbach, The Aspergillus fumigatus septins play pleiotropic roles

306 in septation, conidiation, and cell wall stress, but are dispensable for virulence, Fungal Genetics

307 and Biology, 81 (2015) 41-51.

308 [19] H. Badrane, M.H. Nguyen, J.R. Blankenship, S. Cheng, B. Hao, A.P. Mitchell, C.J. Clancy,

309 Rapid redistribution of phosphatidylinositol-(4,5)-bisphosphate and septins during the Candida

310 albicans response to caspofungin, Antimicrobial agents and chemotherapy, 56 (2012) 4614-

3114624.

312 [20] M.E. da Silva Ferreira, M.R. Kress, M. Savoldi, M.H. Goldman, A. Hartl, T. Heinekamp,

313 A.A. Brakhage, G.H. Goldman, The akuB(KU80) mutant deficient for nonhomologous end 
314 joining is a powerful tool for analyzing pathogenicity in Aspergillus fumigatus, Eukaryotic cell, 5 315 (2006) 207-211.

316 [21] H. Tsai, M.H. Wheeler, Y.C. Chang, K.J. Kwon-Chung, A Developmental Regulated Gene

317 Cluster Involved in Conidial Pigment Biosynthesis in Aspergillus fumigatus, Journal of

318 Bacteriology, 181 (1999) 6469-6477.

319 [22] T.R. Dagenais, D. Chung, S.S. Giles, C.M. Hull, D. Andes, N.P. Keller, Defects in

320 conidiophore development and conidium-macrophage interactions in a dioxygenase mutant of

321 Aspergillus fumigatus, Infect Immun, 76 (2008) 3214-3220.

322 [23] T. Xue, C.K. Nguyen, A. Romans, D.P. Kontoyiannis, G.S. May, Isogenic auxotrophic

323 mutant strains in the Aspergillus fumigatus genome reference strain AF293, Arch Microbiol, 182

324 (2004) 346-353.

325 [24] E. Szewczyk, T. Nayak, C.E. Oakley, E. H., Y. Xiong, N. Taheri-Talesh, S.A. Osmani, B.R.

326 Oakley, Fusion PCR and gene targetin in Aspergillus nidulans, Nature Protocols, 1 (2006) 3111-

3273120.

328 [25] A.A. Soukup, M. Farnoodian, E. Berthier, N.P. Keller, NosA, a transcription factor

329 important in Aspergillus fumigatus stress and developmental response, rescues the germination

330 defect of a laeA deletion, Fungal genetics and biology : FG \& B, 49 (2012) 857-865.

331 [26] P.R. Juvvadi, D. Belina, E.J. Soderblom, M.A. Moseley, W.J. Steinbach, Filamentous 332 fungal-specific septin AspE is phosphorylated in vivo and interacts with actin, tubulin and other 333 septins in the human pathogen Aspergillus fumigatus, Biochemical and biophysical research 334 communications, 431 (2013) 547-553. 
335 [27] CLSI, Reference Method for Broth Dilution Antifungal Susceptibility Testing of

336 Filamentous Fungi: Approved Standard-Second Edition, Clinical and Laboratory Standards

337 Institute, 16 (2008).

338 [28] J.R. Fortwendel, P.R. Juvvadi, N. Pinchai, B.Z. Perfect, J.A. Alspaugh, J.R. Perfect, W.J.

339 Steinbach, Differential effects of inhibiting chitin and 1,3-\{beta\}-D-glucan synthesis in ras and

340 calcineurin mutants of Aspergillus fumigatus, Antimicrobial agents and chemotherapy, 53 (2009)

$341 \quad 476-482$.

342 [29] P.R. Juvvadi, J.R. Fortwendel, L.E. Rogg, W.J. Steinbach, Differential localization patterns

343 of septins during growth of the human fungal pathogen Aspergillus fumigatus reveal novel

344 functions, Biochemical and biophysical research communications, 405 (2011) 238-243.

345 [30] Y. Hernandez-Rodriguez, S. Masuo, D. Johnson, R. Orlando, A. Smith, M. Couto-

346 Rodriguez, M. Momany, Distinct Septin Heteropolymers Co-Exist during Multicellular

347 Development in the Filamentous Fungus Aspergillus nidulans, PloS one, 9 (2014) e92819.

348 [31] I. Sinha, Y.M. Wang, R. Philp, C.R. Li, W.H. Yap, Y. Wang, Cyclin-dependent kinases

349 control septin phosphorylation in Candida albicans hyphal development, Developmental cell, 13

350 (2007) 421-432.

351 [32] C.S.L. Tang, S.I. Reed, Phosphorylation of the Septin Cdc3 in G1 by Cdc28 Kinase Is

352 Essential for Efficient Septin Ring Disassembly, Cell cycle, 1 (2002) 42-29.

353 [33] M. Versele, J. Thorner, Septin collar formation in budding yeast requires GTP binding and

354 direct phosphorylation by the PAK, Cla4, Journal of Cell Biology, 164 (2004) 701-715.

355 [34] P. Wiemann, C.J. Guo, J.M. Palmer, R. Sekonyela, C.C. Wang, N.P. Keller, Prototype of an

356 intertwined secondary-metabolite supercluster, Proceedings of the National Academy of

357 Sciences of the United States of America, 110 (2013) 17065-17070. 
358 [35] U. Garscha, F. Jerneren, D. Chung, N.P. Keller, M. Hamberg, E.H. Oliw, Identification of

359 dioxygenases required for Aspergillus development. Studies of products, stereochemistry, and

360 the reaction mechanism, J Biol Chem, 282 (2007) 34707-34718.

361 [36] K.J. Boyce, H. Chang, C.A. D'Souza, J.W. Kronstad, An Ustilago maydis septin is required

362 for filamentous growth in culture and for full symptom development on maize, Eukaryotic cell, 4

363 (2005) 2044-2056.

364 [37] A.J. Warenda, J.B. Konopka, Septin Function in Candida albicans Morphogenesis,

365 Molecular biology of the cell, 13 (2002) 2732-2746.

366 [38] J.R. Blankenship, S. Cheng, C.A. Woolford, W. Xu, T.M. Johnson, P.D. Rogers, S.

367 Fanning, M.H. Nguyen, C.J. Clancy, A.P. Mitchell, Mutational Analysis of Essential Septins

368 Reveals a Role for Septin-Mediated Signaling in Filamentation, Eukaryotic cell, 13 (2014).

369 [39] J. Romano, G. Nimrod, N. Ben-Tal, Y. Shadkchan, K. Baruch, H. Sharon, N. Osherov,

370 Disruption of the Aspergillus fumigatus ECM33 homologue results in rapid conidial germination,

371 antifungal resistance and hypervirulence, Microbiology, 152 (2006) 1919-1928.

372 [40] D.S. Perlin, Resistance to echinocandin-class antifungal drugs, Drug Resist Updat, 10

373 (2007) 121-130.

374 [41] R.E. Gardiner, P. Souteropoulos, S. Park, D.S. Perlin, Characterization of Aspergillus

375 fumigatus mutants with reduced susceptibility to caspofungin, Medical Mycology, 43 (2005) $376 \quad 299-305$.

377 [42] A. Berepiki, N.D. Read, Septins are important for cell polarity, septation and asexual spore

378 formation in Neurospora crassa and show different patterns of localisation at germ tube tips,

379 PloS one, 8 (2013) e63843. 
380 [43] C. Renz, S. Oeljeklaus, S. Grinhagens, B. Warscheid, N. Johnsson, T. Gronemeyer,

381 Identification of Cell Cycle Dependent Interaction Partners of the Septins by Quantitative Mass

382 Spectrometry, PloS one, 11 (2016) e0148340.

383 [44] M. Ishikawa, T. Ninomiya, H. Akabane, N. Kushida, G. Tsujiuchi, M. Ohyama, S. Gomi, K.

384 Shito, T. Murata, Pseurotin A and its analogues as inhibitors of immunoglobulin E [correction of

385 immunoglobuline E] production, Bioorg Med Chem Lett, 19 (2009) 1457-1460.

386 [45] M. Vodisch, K. Scherlach, R. Winkler, C. Hertweck, H.P. Braun, M. Roth, H. Haas, E.R.

387 Werner, A.A. Brakhage, O. Kniemeyer, Analysis of the Aspergillus fumigatus proteome reveals

388 metabolic changes and the activation of the pseurotin A biosynthesis gene cluster in response to

389 hypoxia, J Proteome Res, 10 (2011) 2508-2524. 


\begin{tabular}{|c|c|c|}
\hline AspGD ID & Protein & Spectral Count \\
\hline \multicolumn{3}{|l|}{ Septins } \\
\hline AFUA_5G08540 & AspA & 329 \\
\hline AFUA_5G03080 & AspC & 415 \\
\hline AFUA_1G08850 & AspD & 394 \\
\hline AFUA_3G07015 & AspE & 40 \\
\hline \multicolumn{3}{|l|}{ Cytoskeleton } \\
\hline AFUA_6G04740 & Act1 & 74 \\
\hline AFUA_2G14990 & TubB & 28 \\
\hline AFUA_1G02550 & Tubulin Alpha subunit & 65 \\
\hline AFUA_1G10910 & Tubulin Beta subunit & 77 \\
\hline \multicolumn{3}{|c|}{ Phosphorylation/dephosphorylation } \\
\hline AFUA_5G05900 & Cla4 & 4 \\
\hline AFUA_6G07980 & $\operatorname{NimX}$ & 7 \\
\hline AFUA_5G09360 & CnaA & 3 \\
\hline AFUA_4G13720 & MpkA & 3 \\
\hline AFUA_1G05800 & MkkA & 9 \\
\hline AFUA_1G09170 & MobB & 5 \\
\hline \multicolumn{3}{|c|}{ Cell Wall Synthesis /Hyphal Growth/Polarity } \\
\hline AFUA_3G14420 & ChsG & 4 \\
\hline AFUA_6G06900 & Rho1 & 20 \\
\hline AFUA_2G07770 & RasB & 14 \\
\hline
\end{tabular}


404 Table 2. Summary of AspB interacting proteins after caspofungin exposure

\begin{tabular}{|c|c|c|}
\hline AspGD ID & Protein & Spectral Count \\
\hline \multicolumn{3}{|c|}{ Secondary Metabolism } \\
\hline AFUA_8G00540 & PsoA PKS-NRPS hybrid synthase & 41 \\
\hline AFUA_5G10120 & NRPS-Like & 3 \\
\hline AFUA_8G00440 & PsoF Mono-oxygenase & 7 \\
\hline \multicolumn{3}{|c|}{ Induced after exposure to antifungal agents } \\
\hline AFUA_1G13330 & ArpB & 4 \\
\hline AFUA_2G03720 & Cyp4 & 3 \\
\hline AFUA_4G10770 & PpoA & 9 \\
\hline
\end{tabular}

406 Table 3 Minimum effective concentration of caspofungin and nikkomycin $\mathbf{Z}$ on Aspergillus

407 fumigatus strains

\begin{tabular}{|c|c|c|}
\hline Strain & Caspofungin $(\boldsymbol{\mu g} / \mathbf{m L})$ & $\begin{array}{c}\text { Nikkomycin } \mathbf{Z} \\
(\boldsymbol{\mu g} / \mathbf{m L})\end{array}$ \\
\hline AF293 & 1 & 2 \\
\hline$\triangle p p o A$ & 0.25 & 1 \\
\hline$O E:: p p o A$ & 2 & 4 \\
\hline
\end{tabular}


Figure 1

A

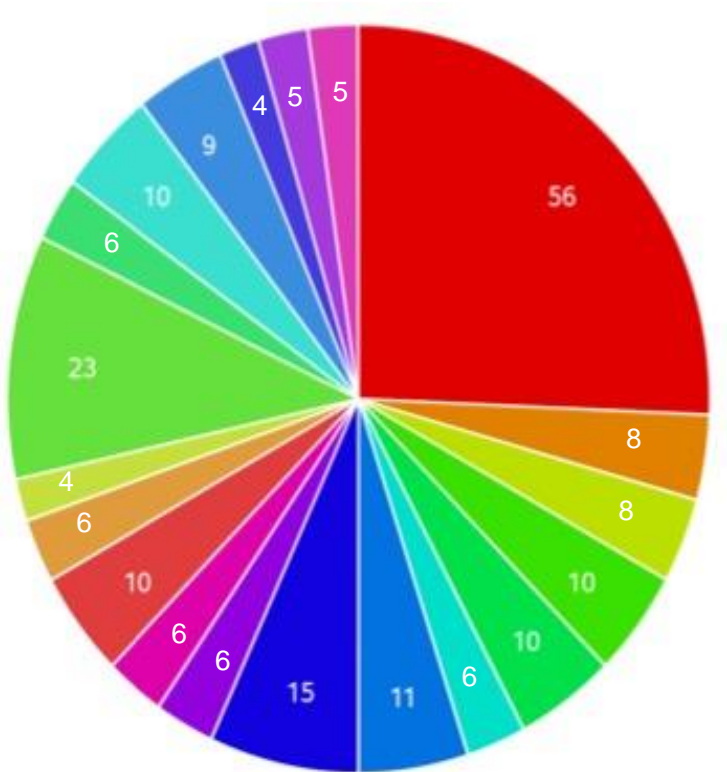

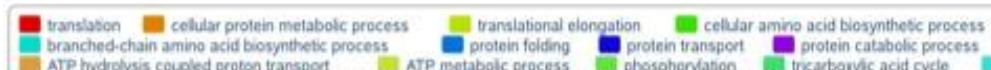

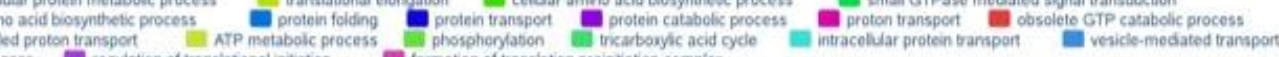

B

Untreated

$(\mathrm{N}=334)$

69
Caspofungin

$(\mathrm{N}=319)$

54

\section{Figure}

(1)

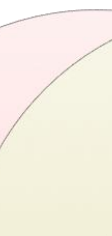

265

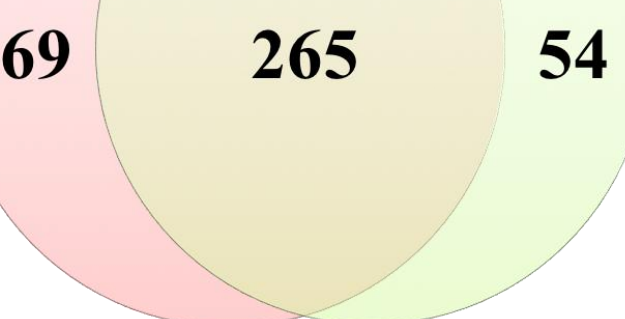


Figure 2

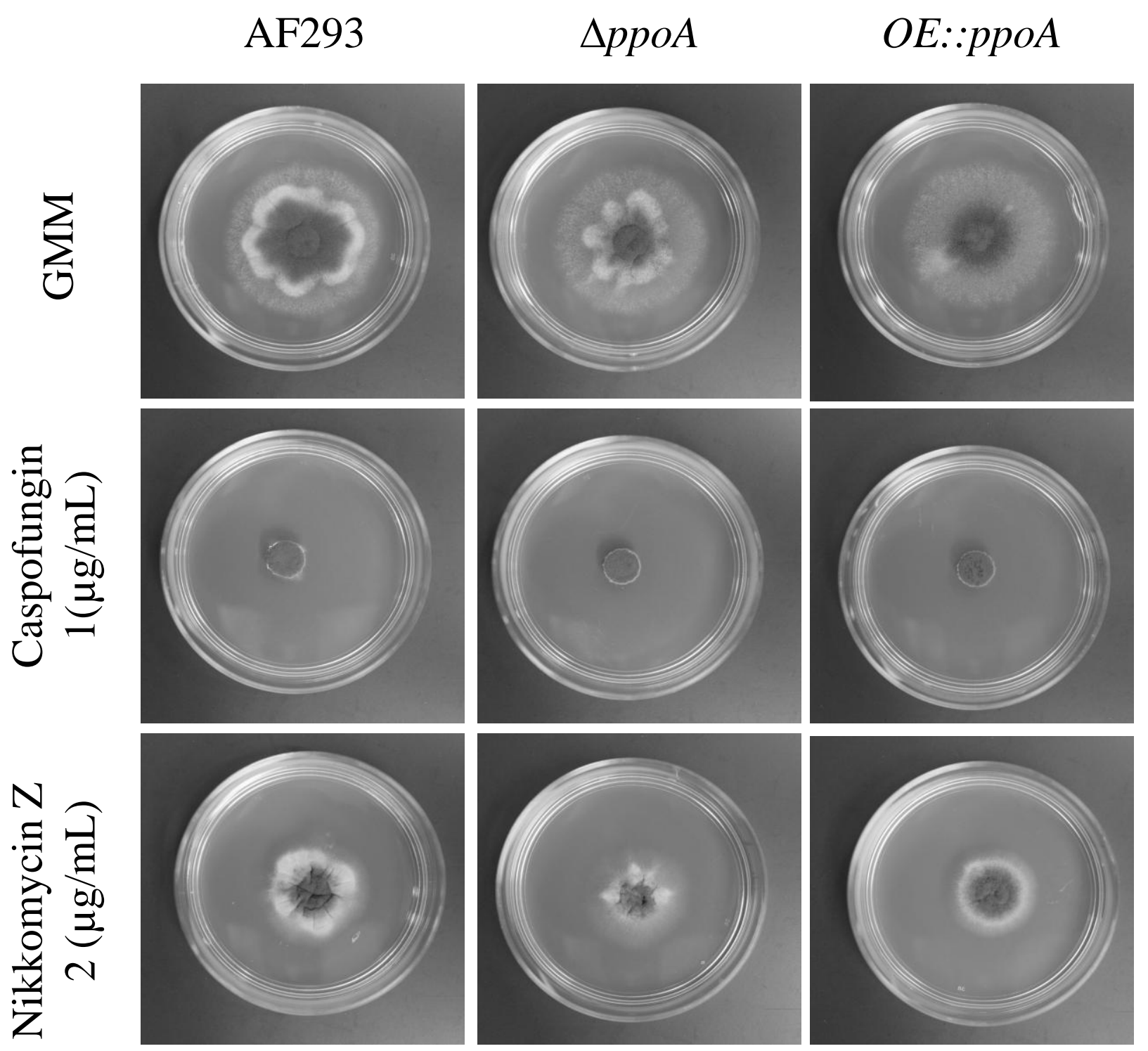

